\title{
A CASE OF AN UNILATERAL TONSILLAR HYPERTROPHY CAUSED BY ACTINOMYCOSIS
}

\author{
Dejan Rašić ${ }^{1}$, Aleksandar Perić ${ }^{1}$, Biserka Vukomanović-Durdević ${ }^{2}$ \\ Military Medical Academy, Belgrade, Serbia: Clinic for Otorhinolaryngology ${ }^{1}$, Institute of Pathology ${ }^{2}$
}

\begin{abstract}
Summary: The purpose of this report was to present an unusual case of an unilateral tonsillar hypertophy caused by actinomycosis. It is an infrequent bacterial infection nowadays. Only five such cases have been reported up to now. Diagnosis is difficult, because there are not specific diagnostic procedures. Medical treatment consists of surgical excision of tonsils and postoperative antibiotic therapy.
\end{abstract}

Key words: Tonsil; Hypertrophy; Unilateral; Actinomycosis

\section{Introduction}

Actinomycosis is a chronic bacterial infection that occurs sporadically throughout the world in humans and animals. Clinically and pathologically, actinomycosis is characterized by swelling, pain, draining sinuses, and a purulent discharge that contains yellow granules („sulfur granules“) (compact bacterial aggregates) (3). The most common agent of actinomycosis in humans is Actinomyces israelii, a commensal saprophyte of the normal flora. It is an anaerobic or microaerophilic Gram-positive organism that grows and reproduces by forming an elongate noncellular mycelium (3). This bacterium may cause infection when it is introduced into the soft tissues by trauma, surgery or another infection (3). The most common site of infection is the cervicofacial region (55\%), followed by the abdominopelvic region (25\%), pulmothoracic region $(15 \%)$, and other locations $(5 \%)(3,8)$.

The role of Actinomyces israeli in the disease of tonsils is not clearly understood. It has been suggested that this infection may indicate an etiological role in tonsillar and adenoidal hypertrophy $(4,5,6,7,10)$. To our knowledge, only five cases of unilateral tonsillar hypertrophy caused by Anktinomyces infection have been reported until now $(4,5,6,7,10)$. Three of them were masquerading as a tumor $(5,6,7)$, and two were pendulous mass attached to the tonsil $(4,10)$.

\section{Case report}

A 14-year-old girl was hospitalized in Military Medical Academy, Belgrade, with two-year history of slow progressive difficulty in respiration and deglutition and increased frequency of episodes of sore throat. The patient reported that the throat infections appeared two years ago, with five or more episodes of tonsillitis per year, followed by high temperature (up to $39^{\circ} \mathrm{C}$ ), dysphagia and cough. On physical examination the patient appeared generally well. Pharyngeal examination showed that the right tonsil was about three times larger than the left one. On pressure, it was soft and tonsillar mucosa seemed normal. The left tonsil seemed atrophic. In the neck region there were palpable bilateral lymph nodes, up to $1 \mathrm{~cm}$ of dimension. Her history was without other illnesses. Nasal, nasopharyngeal and laryngeal examination showed normal findings. Haematological findings were regular. There was absence of a positive throat culture. Biopsy of the right tonsil excluded neoplasma as the etiology. The patient was subjected to surgery and we performed bilateral tonsillectomy under general anesthesia. The both tonsills were dissected out with moderate bleeding. They were labelled and sent separately for histopathological examination. Hematoxylin-eosin (H\&E)-stained tissue sections of the right tonsil demonstrated reactive lymphoid follicular hyperplasia and „sulfur granules“ of Actinomyces in dilated crypts (Fig. 1). Typical sulfur granules were presented as aggregates of filamentous basophillic microorganisms (Fig. 2). The left tonsil demonstrated only low grade of follicular hyperplasia.

After receiving definitive histopathological results, the patient was treated with Penicillin G (1.600.000 I.J. per day) for 10 days. The patient was released on the $14^{\text {th }}$ postoperative day. The postoperative period was normal. At the latest follow-up evaluation (six months postoperatively) she was well and without signs of recurrence.

\section{Discussion}

Cervicofacial actinomycosis, or „lumpy jaw“, is the most common form of this infection, and the disease may deve- 


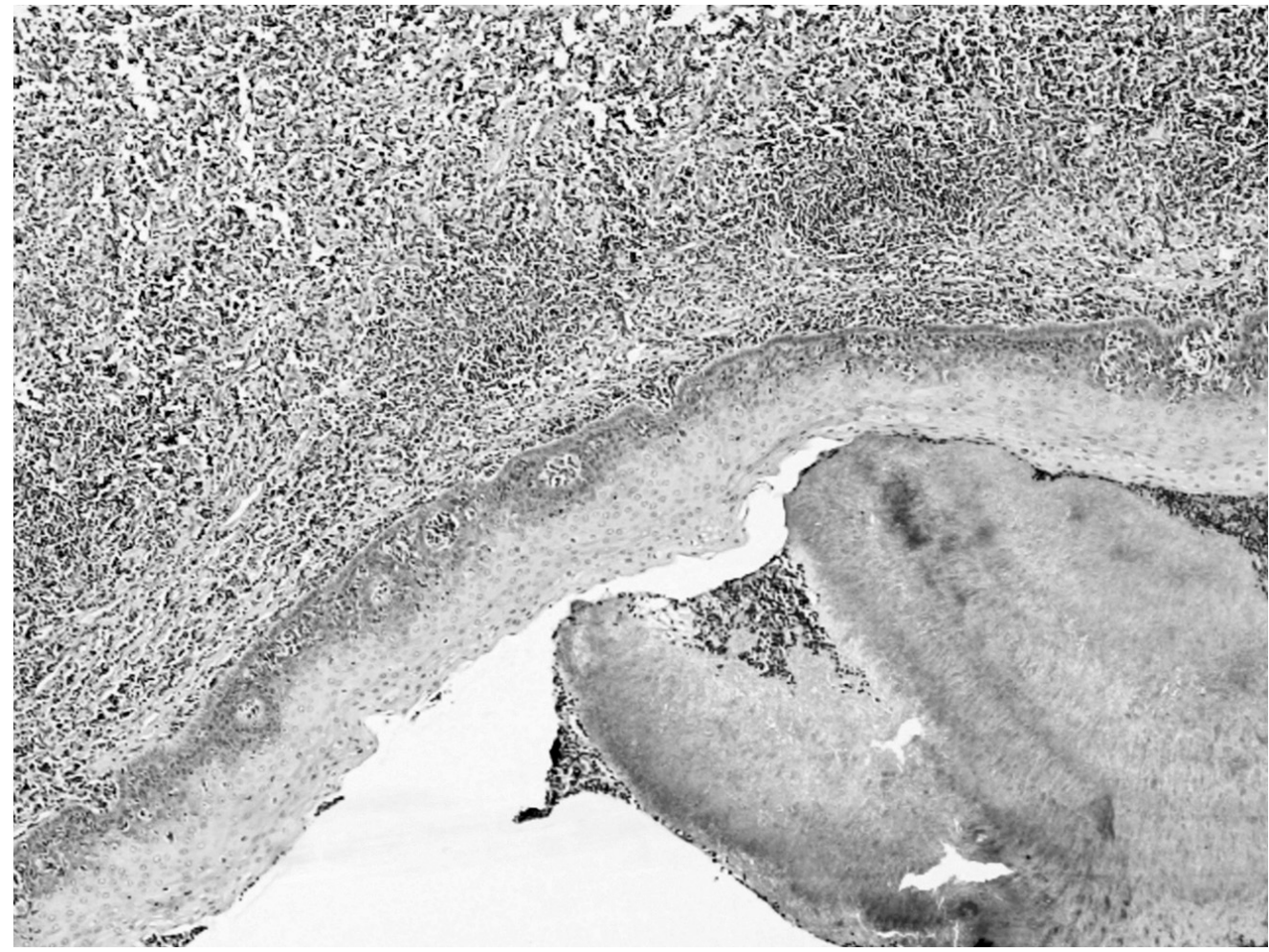

Fig. 1: Tissue section of right tonsil showing „sulfur granule“ of Actinomyces inside the tonsillar crypt and hyperplastic lymphoid follicle inside the tonsillar stroma (H\&E, x 100 magnification).

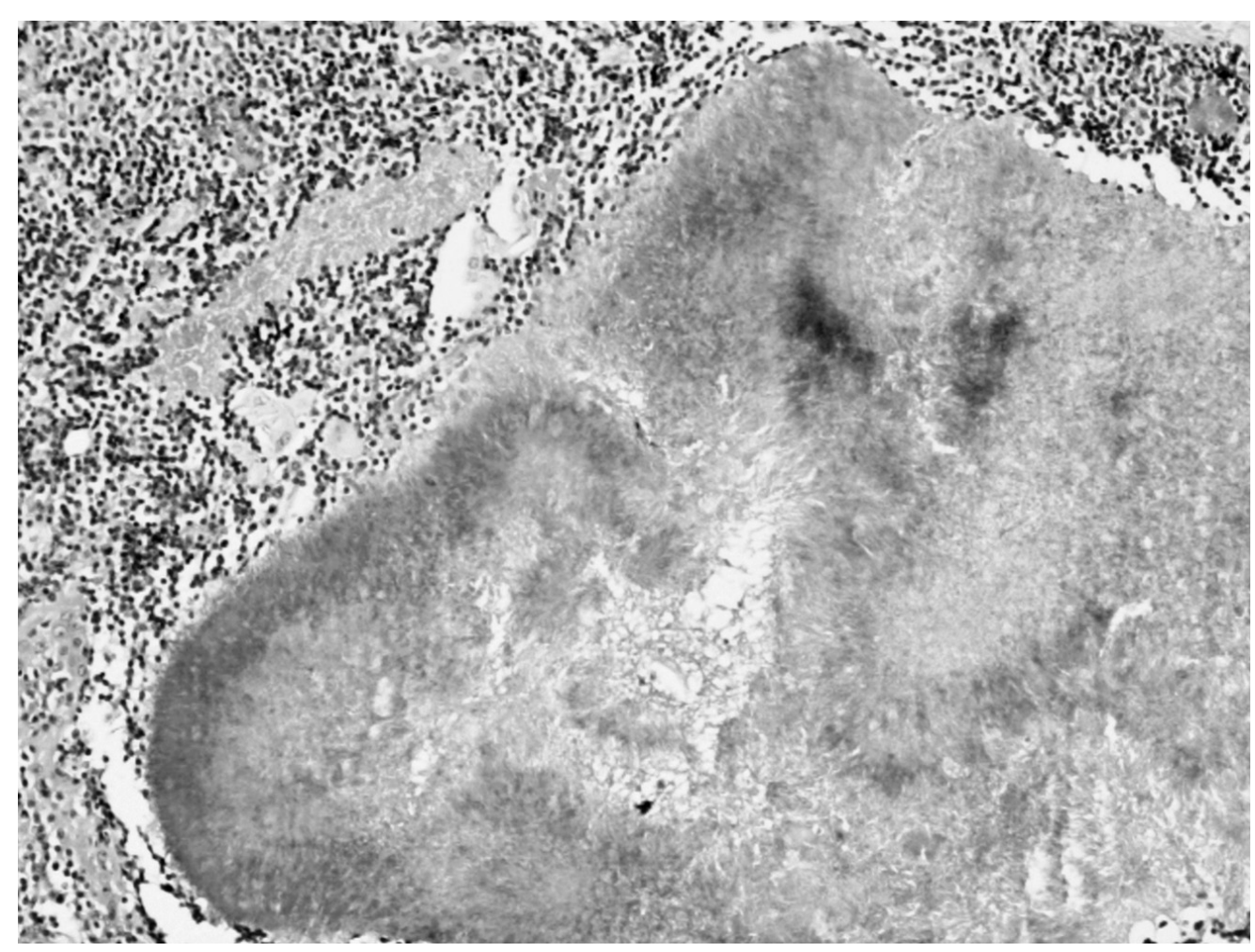

Fig. 2: „Sulfur granule“: aggregates of basophilic, filamentous Actinomyces is located within tonsillar crypt (H\&E, x 200 magnification). 
lop with and without antecedent injury to the oral mucosa (3). Frequently, however, cervicofacial infection follows a dental extraction, or it may complicate dental caries, periodontal diesease or an accidental injury to the oral mucosa (3). Actinomycetes are anaerobes that release proteolytic enzymes. This causes a diminished oxidation-reduction potential and leads to proliferation of the organisms which invades surrounding tissue (9). It is thought that infection of tonsils with pyogenic aerobic and anaerobic organisms may reduce the oxidation-reduction potential in a similar way as described above (9). This may lead to the proliferation and colonisation of the tonsillar crypts by the actinomycotic organisms, presented as aggregates of basophilic filamentous bacterias arranged in a radial spoke-like fashion, the so-called „ray-funfus“ $(3,9)$.

In the more recent studies, the presence of Actinomyces in tonsils has been reported to be from $6.7 \%$ (1) to $28.5 \%$ (2). Results published by Bhargava et al. (2) showed a significant association of actinomycosis and tonsillar hypertrophy. According to Van Lierop et al. (9), there was no correlation found between the presence of tonsillar actinomycosis and recurrent tonsillitis and/or obstructive tonsillar hypertrophy. Actinomycosis being more common in older children, especially those over 5 years of age (9). However, Aydin et al. (1) found that the rate of actinomycosis was significantly higher in adults than in children. Based on the significant association of cryptitis (inflammation of tonsillar crypts) with actinomycosis, cryptitis can be considered a histopathological indicator for tonsillar actinomycosis (1).

Tonsillar actinomycosis is usually bilateral feature. Although actinomycosis can be detected in the tonsil comparatively frequently, there are only several reports indicating that actinomycosis is relevant to unilateral tonsillar hypertrophy. In the differential diagnosis, we should keep in mind that when one tonsil is much larger than the other, the clinician must suspect unusual infections (botryomycosis, nocardiasis) or neoplasia as the etiology $(3,9)$. Mycobacterium tuberculosis, atypical mycobacterium, fungal organism or actinomycosis may all be infections causing unilateral hypertrophy $(3,9)$.

Classical surgical excision is thought to be the definitive treatment for head and neck actinomycosis, and tonsillec- tomy may ultimately be required for tonsillar actinomycosis $(3,7,8,10)$. After the surgical treatment, penicillins given in high doses are the drugs of choice. Other antibiotics, such as tetracycline and chloramphenicol are effective in patients allergic to penicillins $(3,7,8,10)$.

\section{Conclusion}

Actinomycosis as a cause of unilateral tonsillar hapertrophy is rarely seen in clinical practice. Pre-operative investigations (culture throat, biopsy of tonsil, fine-needle aspiration citology) are not effective in making the diagnosis. That's why diagnosis is very difficult and it is definitive only after surgical excision. For successful treatment, postoperative antibiotic therapy is necessary. Why there are several reports about tonsillar actinomycosis? Probably, one of the reason is insufficient recognition among otolaryngologists. The autor's intention is to show one more case of unilateral tonsillar actinomycosis, but also to remind colleagues of this infection in which to think.

\section{References}

1. Aydin A, Erkilic S, Bayazit YA, Kocer NE, Ozer E, Kanlikama M. Relation between actinomycosis and histopathological and clinical features of the palatine tonsils: a comparative study between adult and pediatric patients. Rev Laryngol Otol Rhinol (Bord) 2005;126:95-8

2. Bhargava D, Bhusnurmath B, Sundaram KR, et al. Tonsillar actinomycosis: a clinicopathological study. Acta Tropica 2001;80:163-8.

3. Chandler FW, Connor DH. Actinomycosis. In: Pathology of infectious diseases, Vol 1, Chapter 40. Stamford, Connecticut: Appleton \& Lange;1997:391-6.

4. Hirai S, Akisada T, Yoshihiro T, Take T, Orita Y. A case report of a pendulous tonsil induced by Actinomyces infection. Pract Otol (Kyoto) 1998;(Suppl 96): 146-8.

5. Morikawa I, Shin T, Watanabe H, Matsuo K, Yukizane K. Actinomycosis originating from palatine tonsil and its diagnosis. Otologia Fukuoka 1985;31:382-6.

6. Samant S, Sandoe J, High A, Makura ZG. Actinomycosis mimiking a tonsilla neoplasm in an elderly diabetic patient. Br J Maxillofac Surg 2009;47:417-8.

7. Takasaki K, Kitaoka K, Kaieda S, Hayashi T, Abe K, Takahashi H. A case of actinomycosis causing unilateral tonsillar hypertrophy. Acta Otolaryngol 2006; 126:1001-4

8. Uslu C, Oysu C, Ülkümen B. Coexistence of actinomycosis and sialolithiasis in the submandibular gland. Kulak Burun Bogaz Ihtis Derg 2008;18:257-9.

9. Van Lierop AC, Prescott CAJ, Sinclair-Smith CC. An investigation of the significance of actinomycosis in tonsil disease. Int J Pediatr Otorhinolaryngol 2007; 71:1883-8.

10. Yadav SPS, Chanda R, Gathwala G, Yadav RK. Actinomycosis of tonsil masquerading as tumour in a 12-year old child. Int J Pediatr Otorhinolaryngol 2002; 63:73-5.

Received: 27/01/2010.

Accepted: 05/03/2010.

\section{Corresponding author:}

Dejan Rašić, MD, Clinic for Otorhinolaryngology, Military Medical Academy, Crnotravska 17, 11040 Belgrade, Serbia; e-mail: dejan_rasic@vektor.net 